\title{
Using National Health Insurance Claims Data to Supplement Notifiable Infectious Disease Surveillance System
}

\author{
Heeyoung Lee1, Kwan Lee², Seon-ju Yi¹, Gichan Park1, Hwami Kim¹, SoYoon Min', Jee \\ soo Suh', Young-Man Kim ${ }^{1}$, Soojung Jo' and Daeun Jeong ${ }^{1}$
}

${ }^{1}$ Gyeonggi Infectious Disease Control Center, Seongnam-si, Korea (the Republic of); ${ }^{2}$ Department of Preventive Medicine, Dongguk University College of Medicine, Gyeongju, Korea (the Republic of)

\section{Objective}

This study will determine opportunity of using the National Health Insurance (NHI) claims data for supplemental notifiable infectious disease surveillance system at national or regional levels.

\section{Introduction}

Infectious disease surveillance is very important as an element in public health system in the prevention and control of infectious diseases. Results of the Korean National Notifiable Disease Surveillance System (KNNDSS) has contributed to the reduction of amount of infectious disease. Nevertheless, the "reporting rate" is continuously being debated [1].

The KNNDSS classifies 77 infectious diseases into 6 groups: Group I for those requiring immediate control measures; Group II for vaccine-preventable diseases; Group III for diseases that need routine monitoring; Group IV for emerging diseases in Korea; Group V for parasitic infections; and Group VI for disease that need monitoring outbreaks. Group I - VI diseases are monitored by mandatory surveillance system that requires obligatory reporting on infectious diseases 'without delay' to a district health center [2]

The using the National Health Insurance (NHI) claims data is the important source of information for healthcare service research in South Korea, since South Korea achieves universal coverage of its population. In the aspects of data quality and standard, the sixth revision of the Korean Classification of Disease (KCD-6) has been used in Korea since 2011, and $99.9 \%$ of healthcare providers use to claims to insurers utilizing Electronic Data Interchange transactions. In this respects, NHI claims data is an opportunity as a supplement for NDDSS data.

In this study, we explored the difference between NNDSS data and NHI claim data and determined opportunity and challenges using NHI data for estimation the magnitude of national infectious disease incidence.

\section{Methods}

Cases in NNDSS data and NHI claim data were aggregated yearly from 2011 to 2014. The trends between two data were compared using Spearman's rank correlation coefficient. And also, we classified infectious diseases into four groups according to appearance or trends: (1) disease that there is no incidence in world and Korea, so the use of claims data is difficult, (2) diseases that the trend is coincided or similar between NNDSS data and NHI claim data, (3) diseases that recently the reporting rate increase and it can be used as evaluation index to improve the reporting rate, (4) Etc.

\section{Results}

The First, there were claims for small pox, polio, diphtheria, etc. in NHI data, and they were classified type I. For these, process of error checking of NHI claim data is needed.
Brucellosis $(\mathrm{R}=1.000)$, malaria $(\mathrm{R}=0.943)$, hepatitis $\mathrm{A}(\mathrm{R}=0.943)$, typhoid fever $(\mathrm{R}=0.886)$, etc. were classified type II, but their reporting rate is low, efforts for increase of the reporting rate is required.

Diseases that recently the reporting rate increase, such as chicken pox, mumps, and scarlet fever were classified type III, and it can be used as evaluation index to improve the reporting rate.

\section{Conclusions}

The NHI claims data is the important source of information for healthcare service research in South Korea. Diseases classified type II and III could be used for supplemental NNDSS. Nevertheless, we suggests that comparison between NNDSS data and NHI data for type I and IV is not appropriate.

\section{Keywords}

Korean National Notifiable Disease Surveillance System; Notifiable Disease; National Health Insurance claim data

\section{*Seon-ju Yi}

E-mail: yiseonju@gidcc.or.kr 